\title{
The sirtuin family's role in aging and age-associated pathologies
}

\author{
Jessica A. Hall, John E. Dominy, Yoonjin Lee, and Pere Puigserver
}

Department of Cancer Biology, Dana-Farber Cancer Institute and Department of Cell Biology, Harvard Medical School, Boston, Massachusetts, USA.

\begin{abstract}
The 7 mammalian sirtuin proteins compose a protective cavalry of enzymes that can be invoked by cells to aid in the defense against a vast array of stressors. The pathologies associated with aging, such as metabolic syndrome, neurodegeneration, and cancer, are either caused by or exacerbated by a lifetime of chronic stress. As such, the activation of sirtuin proteins could provide a therapeutic approach to buffer against chronic stress and ameliorate age-related decline. Here we review experimental evidence both for and against this proposal, as well as the implications that isoform-specific sirtuin activation may have for healthy aging in humans.
\end{abstract}

\section{Introduction}

Human life spans have doubled since the 1800s, albeit not without complication. As average life expectancy increases, so does the propensity for aging-associated maladies. Part and parcel with this trend in life span, therefore, has been the quest to manage agerelated disease and extend an individual's healthy life. To date, the only successful intervention to significantly slow the aging process is caloric restriction (CR). CR extends life span in organisms ranging from S. cerevisiae to rodents (1). In addition, CR can delay the onset of numerous diseases associated with aging, such as cancer and diabetes, as possibly evidenced in non-human primates $(2,3)$. This ability of CR to retard the aging process is dependent, at least in part, upon activation of the sirtuin family of proteins. In 1999 it was reported that a sirtuin protein in brewer's yeast, silent information regulator 2 (Sir2), could extend life span in S. cerevisiae (4). Subsequent studies suggested that a similar effect occurred in worms and flies (5), and a large field of research into the age-related biology of sirtuins was cultivated. Although recent reports have questioned the role of sirtuins as direct determinants of longevity (6), the consensus is that they certainly can influence the physiological/pathological quality of life. Herein, we review the role of sirtuins in healthy aging, with particular emphasis on the implication for sirtuin-targeted therapy in protecting humans from age-related physiological decline.

\section{Overview of the mammalian sirtuin family}

There are 7 mammalian sirtuins, SIRT1-7, which are evolutionarily united by their highly conserved $\mathrm{NAD}^{+}$-binding, catalytic domain (7). Despite this commonality, however, a growing body of evidence suggests that their differences may far outweigh their similarities. This is highlighted by distinct expression patterns, catalytic activities, and - ultimately - biological functions. The mammalian sirtuins have discrete subcellular localizations, with a subset of sirtuins residing in predominantly nuclear (SIRT1, SIRT6, and SIRT7), cytosolic (SIRT2), or mitochondrial (SIRT3, SIRT4, and SIRT5) compartments (8). Most sirtuins display protein deacetylase activity, such that they deacetylate internal lysine residues that are acetylated upon their $\varepsilon$-amino groups. Exceptions

Authorship note: Jessica A. Hall, John E. Dominy, and Yoonjin Lee contributed equally to this work.

Conflict of interest: The authors have declared that no conflict of interest exists. Citation for this article: J Clin Invest. 2013;123(3):973-979. doi:10.1172/JCI64094. include SIRT4, which is known only for ADP-ribosyltransferase activity (9), and SIRT5, which has very effective demalonylase and desuccinylase activity and only weak deacetylase activity $(10,11)$.

In the case of acetylation, this reversible post-translational modification quickly manipulates a protein's activity and regulates processes as diverse as metabolic flux and DNA repair (12). The specific activation of sirtuins, therefore, would favor an overall deacetylated protein state in the cell. Interestingly, dietary stress, such as that accompanying fasting or $\mathrm{CR}$, can produce selective protein deacetylation (13-15), although dietary stress can induce protein acetylation as well. A significant portion of diet-induced deacetylation has been shown to occur through the enhanced activity of certain sirtuins (13), suggesting that stress resistance pathways activated by CR may depend, at least in part, on sirtuin deacetylase activity.

Among the mammalian sirtuins, SIRT1 has been the most extensively characterized for its role in aging. Although much of the attention has gone to SIRT1 and its protection from the onset of chronic diseases, its effect on longevity per se is as of yet unconvincing. Indeed, transgenic mice that overexpress SIRT1 have similar life spans as controls (16). In contrast, recent work with SIRT6 suggests that this sirtuin might hold the most potential for actual life span extension. Mice deficient in SIRT6 show severe metabolic defects and have shorter life spans (17), whereas SIRT6overexpressing male mice (but not female mice) have increased life spans (18). Additionally, SIRT3 is the only sirtuin for which there is evidence for an association with human aging; some (but not all) studies have linked polymorphisms in the SIRT3 genomic locus to survival in elderly (19-22). Given the dearth of evidence for clear life span-extending effects for the sirtuin family as a whole, this Review will focus on the disease-ameliorating potential of the sirtuin protein family. The promise of sirtuin-directed therapies can be inferred from data acquired using sirtuin knockout and sirtuin-overexpressing transgenics, as well as data from putative small-molecule sirtuin activators and inhibitors, an area of intense interest and controversy (see ref. 23 for a review).

\section{Disease states}

\section{Metabolic dysfunction}

Declines in basal metabolic rate and physical activity contribute to an elevated incidence of insulin resistance, obesity, and metabolic syndrome with age (24). Activation of pathways that restore 
insulin sensitivity and improve the utilization of glucose and fatty acids would therefore be of benefit to stemming the pathologies associated with age-related metabolic dysfunction.

SIRT1. Many studies have indicated that SIRT1 is an important target for mitigating metabolic dysfunction. SIRT1 levels increase in response to $\mathrm{CR}$, and the enzyme is able to deacetylate a variety of transcription factors and enzymes involved in central metabolism and energy expenditure (see ref. 25 for a review). Key among these factors is PPAR $\gamma$ coactivator- $1 \alpha$ (PGC- $1 \alpha$ ), a transcriptional coactivator that regulates expression of metabolic genes involved in processes such as hepatic gluconeogenesis, fatty acid oxidation, and mitochondrial biogenesis $(26,27)$. Of particular importance to metabolic syndrome, the deacetylation of PGC-1 $\alpha$ by SIRT1 enhances PGC- $1 \alpha$ activity, yielding increased expression of transporters and catabolic enzymes necessary for the uptake and utilization of free fatty acids (28). The process is most evident during exercise, which promotes the utilization of fatty acid in skeletal muscle (29). Consistent with these effects, SIRT1 promotes browning of adipose tissue by increasing the interaction between PPAR $\gamma$ and PRDM16 (30), a potent inducer of PGC1A expression (31).

Several transgenic models have shown that heightened SIRT1 activity protects against the metabolic derangement associated with obesity. More specifically, it was found that mild overexpression of SIRT1 in mice on a high-fat diet (HFD) and in diabetic $d b / d b$ mice causes a significant reduction in blood glucose and plasma insulin levels without significant change in body weight or fat content $(16,32,33)$. A portion of these effects may be due to the ability of SIRT 1 to maintain $\beta$ cell integrity and function. $\beta$ Cell-specific overexpression of SIRT1 markedly improves insulin secretion and glucose tolerance (34). Similarly, pancreatic expression of SIRT1 diminishes $\beta$ cell death that has been induced by the pro-diabetic drug streptozotocin (35). In addition, the protective effects of SIRT 1 may occur through attenuation of inflammatory responses, as SIRT1 overexpression mitigates HFD-induced hepatic steatosis and adipose tissue-specific inflammation $(33,36)$. Conversely, hepatocyte-specific Sirt1 deletion induces hepatic steatosis and inflammation with chronic HFD feeding (37) and an age-dependent onset of hepatic steatosis with normal-diet feeding (38); this suggests that SIRT1 activation might be useful in treating non-alcoholic fatty liver disease, which shows a strong age-dependent onset.

Highlighting the promise for the therapeutic potential of SIRT1 activation are studies proclaiming beneficial effects of putative SIRT1 activators. One such small molecule, the pharmaceutically engineered sirtuin-activating compound SRT1720 $(39,40)$, was shown to improve the overall health and significantly attenuated the premature death of mice fed a HFD (41). Many of the hallmarks of metabolic syndrome such as insulin resistance, hepatic steatosis, and multi-organ inflammation - but not weight gain - were markedly improved by SRT1720 supplementation. In obese humans, 30 days of supplementation with the putative SIRT1 activator resveratrol produced mild to moderate improvements in a number of different clinical parameters including systolic blood pressure, circulating cytokines, intrahepatic fat content, intramyocellular lipid content, and muscle mitochondrial oxidative phosphorylation capacity (42). Nevertheless conflicting results regarding the mechanism of action of these compounds has resulted in much controversy over their true mechanism (see refs. 43, 44 for reviews).

Additionally, some evidence suggests that reduced SIRT1 activity may be desirable for treating certain types of metabolic dys- function. Transient knockdown of hepatic SIRT1 improved whole body insulin sensitivity and reduced elevated hepatic gluconeogenesis in two rodent diabetic models $(45,46)$. In mice with the LDL receptor deleted, whole body overexpression of SIRT1 improved glycemia but promoted hepatic lipid production and secretion and increased the development of atherosclerotic plaques (47). Sirt1 $1^{+/}$ and $\operatorname{Sirt1}^{-/-}$mice were also found to have lower cholesterol levels and blunted responses to LXR agonists in another study (48). These data stress the importance of context and tissue specificity for the potential application of SIRT1-targeted therapy.

SIRT2. Current evidence suggests a role for SIRT2 in regulating adipose tissue development and function. Whereas SIRT2 expression is induced in adipose tissue during CR (49), its expression is significantly blunted in white adipose tissue of obese patients (50). Targets of SIRT2-mediated deacetylation include the FOXO family of transcription factors. SIRT2 deacetylation of FOXO1 leads to its retention in the nucleus and increases FOXO1-mediated repression of the master regulator of adipogenesis, PPARG, culminating in an inhibition of adipocyte differentiation $(51,52)$. Also, cellbased assays have suggested a role for SIRT2 in promoting fatty acid oxidation in a PGC- $1 \alpha$-dependent manner (50).Thus, SIRT2 activation may prove to be protective against obesity, and its role in metabolic homeostasis deserves further exploration.

SIRT3. Of the mitochondrial sirtuins, SIRT3 is the major deacetylase and the best characterized (53). SIRT3 increases in adipose tissue, skeletal muscle, and liver during CR or fasting (13, 54-56), and conversely decreases in response to caloric excess (54, 57-59) or in diabetic models $(60,61)$. As such, SIRT3 functions to activate enzymes important during $\mathrm{CR}$, such as 3-hydroxy-3-methylglutaryl-CoA synthase 2 for generation of ketones (62) and longchain acyl-CoA dehydrogenase for oxidation of long-chain fatty acids (13). SIRT3 also deacetylates and activates components of the electron transport chain, thereby increasing oxidative phosphorylation (57, 58, 63-65). Accordingly, in Sirt3 germline knockout mice, a chronic HFD regimen results in lower energy expenditure, greater adiposity, and higher insulin resistance. Additionally, these mice develop severe hepatosteatosis, increased inflammatory cytokine levels, and higher plasma lipid levels (59). Another study found that SIRT3 deficiency results in glucose intolerance, decreased insulin signaling, and increased oxidative stress in skeletal muscle (60). Of note, patients with a functional SNP in SIRT3, specifically rs11246020, which results in a point mutation of its catalytic domain that decreases activity, have an increased susceptibility to developing metabolic syndrome (59). However, it is still unknown which tissue(s) are directly responsible for mediating the effects of SIRT3, and interest was recently refueled by a report of both muscle-specific and liver-specific Sirt3 ${ }^{-/-}$mice lacking overt metabolic phenotypes (66).

SIRT4. In contrast to SIRT3, hepatic SIRT4 expression declines slightly during CR and increases in genetic models of diabetes (9, $55,67)$. SIRT4 ADP-ribosylates and inactivates glutamate dehydrogenase, which results in decreased insulin release from $\beta$ cells (9). Notably, this negative regulation of SIRT4 on insulin secretion opposes the positive regulation mentioned above with SIRT1. Likewise, SIRT4 negatively regulates fatty acid oxidation and respiration in hepatocytes and myocytes (67). Cumulatively, these data suggest reciprocal coordination, in which SIRT4 antagonizes the metabolically favorable effects of SIRT1 and SIRT3.

SIRT5. SIRT5 is only beginning to be appreciated for its role in metabolism. Carbamoyl phosphate synthetase 1, mitochondrial 
(CPS1), which limits the rate of the urea cycle in detoxification and disposal of ammonia, is a target of SIRT5-mediated deacetylation (68) and desuccinylation (10). As such, although SIRT5-deficient mice were not found to display overt phenotypes (69), elevated blood ammonia was detected during a prolonged fast (68), seemingly due to a lack of CPS1 regulation. Other SIRT5 targets for demalonylation and desuccinylation include additional metabolic enzymes (10), but the biological significance of such modifications remains to be determined. Future efforts will need to explore these targets in order to better define the role of SIRT5 in metabolism and age-related diseases.

SIRT6. Of the nuclear sirtuins, SIRT6 is unique for its specific localization to heterochromatin, where it is best appreciated for its role in histone modification of metabolically relevant genes. Indeed, SIRT6 was discovered to negatively regulate glycolytic gene expression through deacetylation of histone $\mathrm{H} 3$ lysine 9 (H3K9) at their promoters (70). So crucial is this regulation that Sirt6 whole body knockout mice succumb to severe hypoglycemia around 4 weeks of age (17). Similarly, Sirt6 liver-specific knockout mice exhibit increased glycolysis and disruption of glucose homeostasis (71). Interestingly, mice with neural deletion of Sirt6 have normal glycemia but develop adult-onset obesity, again highlighting the importance of tissue specificity (72).

Conversely, SIRT6 overexpression protects from diet-induced obesity, with mice showing increased glucose tolerance and reduced fat accumulation (73). SIRT6 overexpression also highly reduces the IGF-1 signaling pathway, together leading to increased life span in male mice (18). Combined with other non-metabolic roles of SIRT6, including regulation of the inflammatory pathway $(74)$, DNA damage response $(17,75)$, and genomic integrity $(76,77)$, SIRT6 activation provides protection from the onslaught of metabolic dysfunction associated with aging (i.e., diabetes and obesity) and perhaps from aging itself.

\section{Cardiovascular disease}

Age is one of the major risk factors associated with cardiovascular disease. Part of this complex phenomenon is the deterioration of tissues that constitute the heart and its associated vasculature. This deterioration is aggravated by secondary stresses to the cardiovascular system from lifestyle changes (i.e., decreased physical activity), increases in inflammation and oxidative stress, and metabolic disorders (i.e., insulin resistance). Activation of restorative pathways that could intervene at any level of these dysfunctions could theoretically attenuate the progression of agerelated cardiovascular disease.

SIRT1. The ability of SIRT1 to exert cardioprotective benefits is dependent upon the tissue in which it is manipulated and the degree to which its activity is modulated. As mentioned above, whole body augmentation of SIRT1 activity confers resistance to many of the cardiovascular sequelae associated with metabolic syndrome. In the heart, however, the effects of SIRT1 activation are dependent upon the degree of activity increase. Transgenic mouse lines overexpressing SIRT1 at low to moderate levels in a cardiac-specific fashion show reduced myocardial hypertrophy, interstitial fibrosis, and senescence markers such as $\mathrm{p} 15^{\text {INK } 4 \mathrm{~b}}$ and p19ARF (78). Under these same conditions, low-level SIRT1 transgeneity confers increased resistance to oxidative stress (78). On the other hand, transgenic lines possessing high levels of SIRT1 expression have impaired mitochondrial function, lower levels of ATP, and higher levels of oxidative stress (78).
Also, activation of SIRT1 in endothelial tissues may be of benefit in the protection of endothelial cell function with age. Mice with

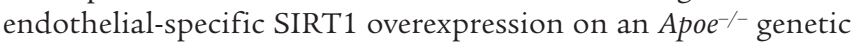
background exhibit attenuated development of atherosclerotic lesions (79). Conversely, SIRT1 insufficiency results in greater foam cell formation and atherosclerotic lesioning (80).

SIRT3. SIRT3 has also been proposed to play a role in agingrelated heart disease. SIRT3-deficient mice show mild cardiac hypertrophy and interstitial fibrosis at baseline and severe cardiac hypertrophy in response to hypertrophic stimuli, whereas SIRT3-overexpressing mice are protected from similar stimuli (81). Although the exact mechanism for the anti-hypertrophic effects of SIRT3 remains unclear, some have suggested that the cardiac pathology of Sirt3 knockout mice stems from an increased activation of the mitochondrial permeability transition pore (mPTP), leading to apoptosis of cardiomyocytes (82). SIRT3 deacetylates and inhibits cyclophilin $\mathrm{D}$, a regulatory component of $\mathrm{mPTP}$, and cardiomyocytes from Sirt3 knockout mice show an age-dependent increase in mitochondrial permeability transition (82). These data suggest that SIRT3 is necessary to prevent mitochondrial dysfunction and cardiac hypertrophy during aging.

SIRT7. Although SIRT7 lacks extensive characterization, the tip of the iceberg seems promising. SIRT7-deficient mice develop a lethal heart hypertrophy, and p53 has been shown to be a target of SIRT7 deacetylation, suggesting that in the absence of SIRT7, p53 activation leads to increased apoptosis in the myocardium (83). Further studies are required to examine whether other SIRT7 targets are related to cardiovascular function.

\section{Cancer}

Based upon statistics from the National Cancer Institute, 54\% of all cancer cases occur in people over the age of 65 . This same cohort of individuals shoulders $70 \%$ of all cancer-related deaths. In this section, we review the literature for a potential role of sirtuin proteins in attenuating cancer susceptibility in the elderly.

SIRT1. SIRT1 expression is elevated in a wide array of cancers, including prostate cancers (84), acute myeloid leukemia (85), colon carcinomas (86), and non-melanoma skin carcinomas (87). This elevated expression suggests that SIRT1 may be a cancer promoter. Inhibition of SIRT1, therefore, could theoretically be a starting point for inducing cell cycle arrest and cause apoptosis in transformed cells. Indeed, some reports indicate that tempering the activity of SIRT1 by either genetic or pharmacological means results in cancer cell death (88-91). Further, the SIRT1 activator SRT1720 was recently reported to promote metastasis in a breast cancer mouse model (92). The proposed mechanisms for these effects are diverse and have been reviewed elsewhere (93).

Other research, however, points to a tumor suppressor role for SIRT1. Certain cancer types, such as mutant breast cancer 1, early onset-mutant (BRCA1) breast cancer cells, contain significantly lower levels of SIRT1 expression than BRCA1 wild-type cells; restoration of SIRT1 levels in these cells results in inhibition of tumor growth (94). Overexpression of SIRT1 has also been shown to reduce the growth rate of fibrosarcoma cells in a tissue allograft model (95). In other mouse models of cancer, SIRT1 can protect against the development of intestinal tumors in a beta-catenindriven colon cancer model (96), sarcomas, lymphomas, teratomas, and carcinomas arising from deletion of p53 (97), HFD-induced hepatocarcinomas (98), and age-associated spontaneous tumor development (98). It remains to be seen what cellular determi- 


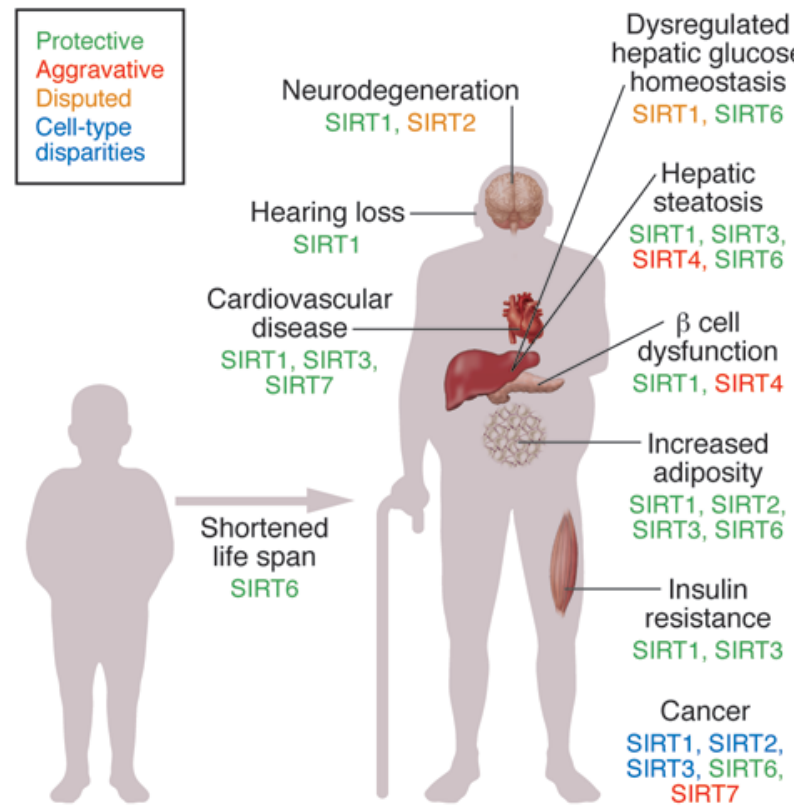

nants underlie SIRT1 being a tumor suppressor under some circumstances and an oncogenic promoter under others.

SIRT2. Mounting evidence implies a role for SIRT2 in cancer. SIRT2 is well appreciated for its regulation of cell cycle progression (99-101) and chromatin assembly $(102,103)$. These roles are best showcased in Sirt2 knockout mice, in which SIRT2 deficiency leads to tumorigenesis due to failed mitosis complex regulation and disrupted genomic integrity (104). However, others have shown that pharmacological inhibition of SIRT2 delays tumor growth through activation of p53 $(91,105)$. Further studies will be required to flesh out these conflicting results and determine the therapeutic potential of SIRT2-specific targeting in cancer.

SIRT3. Several studies indicate that SIRT3 is a tumor suppressor (see ref. 106 for review). Important among these are the findings that SIRT3-deficient mice develop mammary tumors, and embryonic fibroblasts from these mice exhibit a malignancy-prone phenotype with increased stress-induced superoxide levels and genomic instability (64). These cellular defects can be rescued by the expression of SOD2, a target of SIRT3 deacetylation that is involved in the detoxification of reactive oxygen species $(107,108)$. Coinciding with animal data, SIRT3 expression is decreased in many human cancers, with loss of at least one copy of SIRT3 occurring in $40 \%$ of human breast and ovarian cancers $(64,109)$. Nonetheless, there are also reports that SIRT3 overexpression promotes survival and enhances tumorigenesis, such as with oral cancer, emphasizing the importance of tumor type (reviewed in ref. 110).

SIRT6. Although the effect of SIRT6 on tumor formation and growth is a topic still in its infancy, current evidence suggests that SIRT6 is important in the preservation of genomic integrity upon DNA damage and stress stimuli $(76,77,111)$. Thus, in addition to metabolic defects observed in Sirt6 knockout mice, these mice also have an impaired DNA damage response (17), including genomic instability upon irradiation. And although Sirt6 transgenic mice do not show a significant decrease in age-associated tumor formation (18), SIRT6 overexpression might still serve a protective role against tumorigenesis, and this avenue deserves further exploration.

\section{Figure 1}

Anticipated effect of isoform-specific sirtuin activation on various agerelated diseases. Based on the current literature, this figure depicts the potential outcomes associated with individual sirtuin activation (SIRT1-7). The sirtuin isoforms with substantial data indicating either a protective or aggravative role for specific age-related diseases are indicated. There are specific disease states in which there is significant uncertainty in the literature as to whether activation or inhibition of the sirtuin is more beneficial, and these instances are thus classified as "disputed." In the case of cancer, the tumor-suppressor role of certain sirtuin proteins is dependent on cell and tissue type.

SIRT7. An additional deacteylation target of SIRT7, H3K18, was recently identified (112). Importantly, hypoacetylation at H3K18 has been linked to aggressive cancer phenotypes (113). Indeed, SIRT7 knockdown reduces tumor growth of human cancer cell xenografts in mice (112). It will be important for future studies to analyze Sirt7 knockout mice for their resistance to tumor growth.

\section{Neurodegeneration}

Although the body's organ systems experience a general decline in function with age, perhaps the most emotionally and physically devastating is the decline associated with the CNS. Impaired CNS function, with its effects on cognition, memory, hearing, balance, and motor control, can lead to a rapid loss of quality of life. The ability of sirtuins to ameliorate CNS-specific disorders is still a very inchoate area of investigation, yet its initial findings offer significant promise.

SIRT1. Progress in understanding the neurobiological benefits of SIRT1 has been focused on its ability to alter animal models of different human CNS diseases. In mouse models of Alzheimer's disease, brain-specific knockout of Sirt1 caused a significant elevation in $\beta$-amyloid plaques and reactive gliosis (114). On the other hand, overexpression of SIRT1 caused a decrease in these parameters, possibly due to deacetylation by SIRT1 of retinoic acid receptor- $\beta$ (114). Also, SIRT1 conferred neuroprotection in three different mouse models of Huntington's disease $(115,116)$. Brainspecific deletion of SIRT1 exacerbated the neurotoxicity associated with mutant huntingtin protein, whereas overexpression of SIRT1 attenuated the toxicity. The neuroprotective effect of SIRT1 in Huntington's disease models appears to be mediated by the activation of multiple targets, including CREB, CREB regulated transcription coactivator 1, and FOXO3a. In a mouse model of Parkinson's disease, overexpression of SIRT1 reduced $\alpha$-synuclein aggregates, lessened gliosis, and attenuated lethality (117). Knockout of Sirt1, however, significantly increased aggregates, worsened gliosis, and hastened mortality (117). Deacetylation and activation of the heat shock factor HSF1 appears to be the major mechanism 
whereby SIRT1 confers protection against this mouse model of Parkinson's disease. Lastly, in a mouse model of injury-induced axonal degeneration, $\mathrm{NAD}^{+}$biosynthesis and its support of SIRT1 activity was shown to be essential in preventing axonal loss following axonal transection $(118,119)$.

SIRT2. Inhibition of SIRT2 by pharmacological and genetic means in invertebrate and cell culture models has suggested potential neuroprotective benefits. Two such studies demonstrated reduction of SIRT2 to be protective in fly and worm models of Huntington's disease by increasing photoreceptor neuron survival (120) and decreasing sterol biosynthesis (121). Partial rescue of toxicity was also observed in a Drosophila model of Parkinson's disease (122). Unfortunately, however, Sirt2 knockout mice lacked a remarkable phenotype related to neurodegeneration (123), calling into question the effect of SIRT2 on neuronal health in mammals. Interestingly, data from mice with Schwann cell-specific Sirt2 deletion or overexpression have revealed a role for SIRT2 in proper myelination of the peripheral nervous system, with implications for SIRT2 in demyelinating neuropathies (124).

SIRT3. Age-related hearing loss is a common disorder of the elderly that involves oxidative damage to neurons and ciliated cells of the cochlea. A recent study established that the ability of CR to slow the progression of age-related hearing loss is SIRT3 dependent (125). The authors suggested that SIRT3 mediates this effect through deacetylation of isocitrate dehydrogenase 2 and protection of oxidative stress-induced cell death. Whether SIRT3 exerts protection in other neuronal cell types remains to be seen.

\section{Conclusion}

Sirtuin proteins bolster stress resistance of mammalian cells by virtue of their ability to remodel metabolism, alter inflammatory responses, and enhance the ability to cope with oxidative species. Because many of these same pathways are pathologically altered in the aged, activation of sirtuins represents a feasible means for attenuating age-related decline in physiological function (see Figure 1). This directive is in part supported by promising preliminary studies with putative SIRT1 activators, such as SRT1720.
However, several major challenges need to be addressed before intelligent formulations of drugs can be made. The first is a more careful dissection of each sirtuin's ability to ameliorate a particular pathophysiological state. Currently, there is little standardization of the disease models that are being employed to study sirtuin therapeutic biology, leading to contradictory reports with limited cross-validation of the findings. Future directions may also help discern whether there is cooperation or antagonism among the sirtuin paralogs to resolve age-related diseases. The second challenge is to more thoroughly reconcile whether there are any negative effects associated with chronic sirtuin activation, particularly in older cohorts. The vast majority of reports suggest that there are only benefits to be accrued from their activation, but a more skeptical approach may be warranted. The third major challenge is the development of more isoform-specific activators and/or inhibitors. The most effective class of sirtuin activators has only been reported for SIRT1. Nevertheless, as we learn more about the biology of other sirtuins, it has become apparent that activation of the paralogs of SIRT1 may be more beneficial under certain circumstances. In conclusion, although the optimism surrounding the therapeutic potential of sirtuins in treating age-related conditions should be cautious, the trend in encouraging literature suggests that good things are yet to come from the sirtuin family.

\section{Acknowledgments}

J.A. Hall is supported by an American Heart Association predoctoral fellowship. J.E. Dominy is supported in part by an NIH National Research Service Award Kirschstein fellowship. Y. Lee is funded by a scholarship from Ewha Womans University. P. Puigserver has received support from the Dana-Farber Cancer Institute, American Diabetes Association, Department of Defense, and NIH/NIDDK (grant RO1069966).

Address correspondence to: Pere Puigserver, Dana-Farber Cancer Institute, 450 Brookline Ave., CLSB-11144, Boston, Massachusetts 02215, USA. Phone: 617.582.7977; Fax: 617.632.5363; E-mail: pere_puigserver@dfci.harvard.edu.
1. Koubova J, Guarente L. How does calorie restriction work? Genes Dev. 2003;17(3):313-321.

2. Mattison JA, et al. Impact of caloric restriction on health and survival in rhesus monkeys from the NIA study. Nature. 2012;489(7415):318-321.

3. Colman RJ, et al. Caloric restriction delays disease onset and mortality in rhesus monkeys. Science. 2009;325(5937):201-204.

4. Kaeberlein M, McVey M, Guarente L. The SIR2/3/4 complex and SIR2 alone promote longevity in Saccharomyces cerevisiae by two different mechanisms. Genes Dev. 1999;13(19):2570-2580.

5. Chen D, Guarente L. SIR2: a potential target for calorie restriction mimetics. Trends Mol Med. 2007; 13(2):64-71.

6. Burnett C, et al. Absence of effects of Sir2 overexpression on lifespan in C. elegans and Drosophila. Nature. 2011;477(7365):482-485.

7. Frye RA. Phylogenetic classification of prokaryotic and eukaryotic Sir2-like proteins. Biochem Biophys Res Commun. 2000;273(2):793-798.

8. Houtkooper RH, Pirinen E, Auwerx J. Sirtuins as regulators of metabolism and healthspan. Nat Rev Mol Cell Biol. 2012;13(4):225-238.

9. Haigis MC, et al. SIRT4 inhibits glutamate dehydrogenase and opposes the effects of calorie restriction in pancreatic beta cells. Cell. 2006;126(5):941-954.

10. Du J, et al. Sirt5 is a NAD-dependent protein lysine demalonylase and desuccinylase. Science.
2011;334(6057):806-809.

11. Peng $\mathrm{C}$, et al. The first identification of lysine malonylation substrates and its regulatory enzyme. Mol Cell Proteomics. 2011;10(12):M111.012658.

12. Wellen KE, Thompson CB. A two-way street: reciprocal regulation of metabolism and signalling. Nat Rev Mol Cell Biol. 2012;13(4):270-276.

13. Hirschey MD, et al. SIRT3 regulates mitochondrial fatty-acid oxidation by reversible enzyme deacetylation. Nature. 2010;464(7285):121-125.

14. Kim SC, et al. Substrate and functional diversity of lysine acetylation revealed by a proteomics survey. Mol Cell. 2006;23(4):607-618.

15. Yang L, et al. The fasted/fed mouse metabolic acetylome: N6-acetylation differences suggest acetylation coordinates organ-specific fuel switching. J Proteome Res. 2011;10(9):4134-4149.

16. Herranz D, et al. Sirt 1 improves healthy ageing and protects from metabolic syndrome-associated cancer. Nat Commun. 2010;1:3.

17. Mostoslavsky R, et al. Genomic instability and aging-like phenotype in the absence of mammalian SIRT6. Cell. 2006;124(2):315-329.

18. Kanfi Y, et al. The sirtuin SIRT6 regulates lifespan in male mice. Nature. 2012;483(7388):218-221.

19. Bellizzi D, et al. Characterization of a bidirectional promoter shared between two human genes related to aging: SIRT3 and PSMD13. Genomics. 2007; 89(1):143-150.
20. Bellizzi D, et al. A novel VNTR enhancer within the SIRT3 gene, a human homologue of SIR2, is associated with survival at oldest ages. Genomics. 2005;85(2):258-263.

21. Rose G, et al. Variability of the SIRT3 gene, human silent information regulator Sir2 homologue, and survivorship in the elderly. Exp Gerontol. 2003; 38(10):1065-1070.

22. Lescai F, et al. Human longevity and 11 p15.5: a study in 1321 centenarians. Eur J Hum Genet. 2009; 17(11):1515-1519.

23. Dittenhafer-Reed KE, Feldman JL, Denu JM. Catalysis and mechanistic insights into sirtuin activation. Chembiochem. 2011;12(2):281-289.

24. Grinker JA, Tucker K, Vokonas PS, Rush D. Body habitus changes among adult males from the normative aging study: relations to aging, smoking history and alcohol intake. Obes Res. 1995;3(5):435-446.

25. Liang F, Kume S, Koya D. SIRT1 and insulin resistance. Nat Rev Endocrinol. 2009;5(7):367-373.

26. Dominy JE, Gerhart-Hines Z, Puigserver P. Nutrient-dependent acetylation controls basic regulatory metabolic switches and cellular reprogramming. Cold Spring Harb Symp Quant Biol. 2011;76:203-209.

27. Gerhart-Hines Z, et al. The cAMP/PKA pathway rapidly activates SIRT1 to promote fatty acid oxidation independently of changes in $\mathrm{NAD}(+)$. Mol Cell. 2011;44(6):851-863.

28. Gerhart-Hines Z, et al. Metabolic control of mus- 
cle mitochondrial function and fatty acid oxidation through SIRT1/PGC-1alpha. EMBO J. 2007; 26(7):1913-1923.

29. Canto C, et al. AMPK regulates energy expenditure by modulating NAD + metabolism and SIRT 1 activity. Nature. 2009;458(7241):1056-1060.

30. Qiang $\mathrm{L}$, et al. Brown remodeling of white adipose tissue by SirT1-dependent deacetylation of Ppargamma. Cell. 2012;150(3):620-632.

31. Kajimura S, et al. Regulation of the brown and white fat gene programs through a PRDM16/ CtBP transcriptional complex. Genes Dev. 2008; 22(10):1397-1409.

32. Banks AS, et al. SirT1 gain of function increases energy efficiency and prevents diabetes in mice. Cell Metab. 2008;8(4):333-341.

33. Pfluger PT, Herranz D, Velasco-Miguel S, Serrano M, Tschop MH. Sirt1 protects against high-fat dietinduced metabolic damage. Proc Natl Acad Sci US A. 2008;105(28):9793-9798.

34. Moynihan KA, et al. Increased dosage of mammalian Sir2 in pancreatic beta cells enhances glucosestimulated insulin secretion in mice. Cell Metab. 2005;2(2):105-117.

35. Tang MM, et al. Intra-arterial targeted islet-specific expression of Sirt1 protects beta cells from streptozotocin-induced apoptosis in mice. Mol Ther. 2011; 19(1):60-66.

36. Gillum MP, et al. SirT1 regulates adipose tissue inflammation. Diabetes. 2011;60(12):3235-3245.

37. Purushotham A, Schug TT, Xu Q, Surapureddi S, Guo X, Li X. Hepatocyte-specific deletion of SIRT1 alters fatty acid metabolism and results in hepatic steatosis and inflammation. Cell Metab. 2009;9(4):327-338.

38. Wang RH, Li C, Deng CX. Liver steatosis and increased ChREBP expression in mice carrying a liver specific SIRT1 null mutation under a normal feeding condition. Int J Biol Sci. 2010;6(7):682-690.

39. Milne JC, et al. Small molecule activators of SIRT1 as therapeutics for the treatment of type 2 diabetes. Nature. 2007;450(7170):712-716.

40. Feige JN, et al. Specific SIRT1 activation mimics low energy levels and protects against diet-induced metabolic disorders by enhancing fat oxidation. Cell Metab. 2008;8(5):347-358.

41. Minor RK, et al. SRT1720 improves survival and healthspan of obese mice. Sci Rep. 2011;1:70

42. Timmers $S$, et al. Calorie restriction-like effects of 30 days of resveratrol supplementation on energy metabolism and metabolic profile in obese humans Cell Metab. 2011;14(5):612-622.

43. Baur JA, Ungvari Z, Minor RK, Le Couteur DG, de Cabo R. Are sirtuins viable targets for improving healthspan and lifespan? Nat Rev Drug Discov. 2012;11(6):443-461.

44. Blum CA, Ellis JL, Loh C, Ng PY, Perni RB, Stein RL. SIRT1 modulation as a novel approach to the treatment of diseases of aging. J Med Chem. 2011; 54(2):417-432.

45. Erion DM, et al. SirT1 knockdown in liver decreases basal hepatic glucose production and increases hepatic insulin responsiveness in diabetic rats. Proc Natl Acad Sci U S A. 2009;106(27):11288-11293.

46. Rodgers JT, Puigserver P. Fasting-dependent glucose and lipid metabolic response through hepatic sirtuin 1. Proc Natl Acad Sci U S A. 2007;104(31):12861-12866

47. Qiang L, Lin HV, Kim-Muller JY, Welch CL, Gu W, Accili D. Proatherogenic abnormalities of lipid metabolism in SirT1 transgenic mice are mediated through Creb deacetylation. Cell Metab. 2011;14(6):758-767.

48. Li X, Zhang S, Blander G, Tse JG, Krieger M, Guarente L. SIRT1 deacetylates and positively regulates the nuclear receptor LXR. Mol Cell. 2007;28(1):91-106.

49. Wang F, Nguyen M, Qin FX, Tong Q. SIRT2 deacetylates FOXO3a in response to oxidative stress and caloric restriction. Aging Cell. 2007;6(4):505-514.

50. Krishnan J, et al. Dietary obesity-associated Hif1al- pha activation in adipocytes restricts fatty acid oxidation and energy expenditure via suppression of the Sirt2-NAD+ system. Genes Dev. 2012;26(3):259-270.

51. Jing E, Gesta S, Kahn CR. SIRT2 regulates adipocyte differentiation through FoxO1 acetylation/ deacetylation. Cell Metab. 2007;6(2):105-114.

52. Wang F, Tong Q. SIRT2 suppresses adipocyte differentiation by deacetylating FOXO1 and enhancing FOXO1's repressive interaction with PPARgamma. Mol Biol Cell. 2009;20(3):801-808.

53. Lombard DB, Tishkoff DX, Bao J. Mitochondrial sirtuins in the regulation of mitochondrial activity and metabolic adaptation. Handb Exp Pharmacol. 2011;206:163-188.

54. Palacios OM, et al. Diet and exercise signals regulate SIRT3 and activate AMPK and PGC-1alpha in skeletal muscle. Aging (Albany NY). 2009;1(9):771-783

55. Schwer B, et al. Calorie restriction alters mitochondrial protein acetylation. Aging Cell. 2009;8(5):604-606.

56. Shi T, Wang F, Stieren E, Tong Q. SIRT3, a mitochondrial sirtuin deacetylase, regulates mitochondrial function and thermogenesis in brown adipocytes. J Biol Chem. 2005;280(14):13560-13567.

57 . Bao J, et al. SIRT3 is regulated by nutrient excess and modulates hepatic susceptibility to lipotoxicity. Free Radic Biol Med. 2010;49(7):1230-1237.

58 . Kendrick AA, et al. Fatty liver is associated with reduced SIRT3 activity and mitochondrial protein hyperacetylation. Biochem J. 2011;433(3):505-514.

59. Hirschey MD, et al. SIRT3 deficiency and mitochondrial protein hyperacetylation accelerate the development of the metabolic syndrome. Mol Cell. 2011;44(2):177-190.

60. Jing E, et al. Sirtuin-3 (Sirt3) regulates skeletal muscle metabolism and insulin signaling via altered mitochondrial oxidation and reactive oxygen species production. Proc Natl Acad Sci U S A. 2011; 108(35):14608-14613.

61. Yechoor VK, et al. Distinct pathways of insulin-regulated versus diabetes-regulated gene expression: an in vivo analysis in MIRKO mice. Proc Natl Acad Sci U S A. 2004;101(47):16525-16530.

62. Shimazu T, et al. SIRT3 deacetylates mitochondrial 3-hydroxy-3-methylglutaryl CoA synthase 2 and regulates ketone body production. Cell Metab. 2010;12(6):654-661.

63. Ahn $\mathrm{BH}$, et al. A role for the mitochondrial deacetylase Sirt3 in regulating energy homeostasis. Proc Natl Acad Sci U S A. 2008;105(38):14447-14452.

64. Kim HS, et al. SIRT3 is a mitochondria-localized tumor suppressor required for maintenance of mitochondrial integrity and metabolism during stress. Cancer Cell. 2010;17(1):41-52.

65. Cimen H, Han MJ, Yang Y, Tong Q, Koc H, Koc EC. Regulation of succinate dehydrogenase activity by SIRT3 in mammalian mitochondria. Biochemistry. 2010;49(2):304-311.

66. Fernandez-Marcos PJ, et al. Muscle or liver-specific Sirt3 deficiency induces hyperacetylation of mitochondrial proteins without affecting global metabolic homeostasis. Sci Rep. 2012;2:425.

67. Nasrin N, et al. SIRT4 regulates fatty acid oxidation and mitochondrial gene expression in liver and muscle cells. J Biol Chem. 2010;285(42):31995-32002.

68. Nakagawa T, Lomb DJ, Haigis MC, Guarente L. SIRT5 Deacetylates carbamoyl phosphate synthetase 1 and regulates the urea cycle. Cell. 2009;137(3):560-570.

69. Lombard DB, et al. Mammalian Sir2 homolog SIRT3 regulates global mitochondrial lysine acetylation. Mol Cell Biol. 2007;27(24):8807-8814.

70. Zhong L, et al. The histone deacetylase Sirt6 regulates glucose homeostasis via Hif1alpha. Cell. 2010;140(2):280-293.

71. Kim HS, et al. Hepatic-specific disruption of SIRT6 in mice results in fatty liver formation due to enhanced glycolysis and triglyceride synthesis. Cell Metab. 2010;12(3):224-236.

72. Schwer B, et al. Neural sirtuin 6 (Sirt6) ablation attenuates somatic growth and causes obesity. Proc Natl Acad Sci US A. 2010;107(50):21790-21794.

73. Kanfi Y, et al. SIRT6 protects against pathological damage caused by diet-induced obesity. Aging Cell. 2010;9(2):162-173

74. Kawahara TL, et al. SIRT6 links histone H3 lysine 9 deacetylation to NF-kappaB-dependent gene expression and organismal life span. Cell. 2009; 136(1):62-74.

75. Kaidi A, Weinert BT, Choudhary C, Jackson SP. Human SIRT6 promotes DNA end resection through CtIP deacetylation. Science. 2010; 329(5997):1348-1353.

76. Yang B, Zwaans BM, Eckersdorff M, Lombard DB. The sirtuin SIRT6 deacetylates H3 K56Ac in vivo to promote genomic stability. Cell Cycle. 2009;8(16):2662-2663.

77. Michishita E, et al. Cell cycle-dependent deacetylation of telomeric histone $\mathrm{H} 3$ lysine K56 by human SIRT6. Cell Cycle. 2009;8(16):2664-2666.

78. Alcendor RR, et al. Sirt1 regulates aging and resistance to oxidative stress in the heart. Circ Res. 2007;100(10):1512-1521.

79. Zhang QJ, et al. Endothelium-specific overexpression of class III deacetylase SIRT1 decreases atherosclerosis in apolipoprotein E-deficient mice. Cardiovasc Res. 2008;80(2):191-199.

80. Stein S, et al. SIRT1 decreases Lox-1-mediated foam cell formation in atherogenesis. Eur Heart J. 2010;31(18):2301-2309.

81. Sundaresan NR, Gupta M, Kim G, Rajamohan SB, Isbatan A, Gupta MP. Sirt3 blocks the cardiac hypertrophic response by augmenting Foxo3adependent antioxidant defense mechanisms in mice. J Clin Invest. 2009;119(9):2758-2771.

82. Hafner AV, et al. Regulation of the MPTP by SIRT3mediated deacetylation of CypD at lysine 166 suppresses age-related cardiac hypertrophy. Aging (Albany NY). 2010;2(12):914-923.

83. Vakhrusheva O, et al. Sirt7 increases stress resistance of cardiomyocytes and prevents apoptosis and inflammatory cardiomyopathy in mice. Circ Res. 2008;102(6):703-710.

84. Huffman DM, et al. SIRT1 is significantly elevated in mouse and human prostate cancer. Cancer Res. 2007;67(14):6612-6618

85. Bradbury CA, et al. Histone deacetylases in acute myeloid leukaemia show a distinctive pattern of expression that changes selectively in response to deacetylase inhibitors. Lenkemia. 2005; 19(10):1751-1759.

86. Stunkel W, et al. Function of the SIRT1 protein deacetylase in cancer. Biotechnol J. 2007:2(11):1360-1368.

87. Hida Y, Kubo Y, Murao K, Arase S. Strong expression of a longevity-related protein, SIRT1, in Bowen's disease. Arch Dermatol Res. 2007;299(2):103-106.

88. Ford J, Jiang M, Milner J. Cancer-specific functions of SIRT1 enable human epithelial cancer cell growth and survival. Cancer Res. 2005;65(22):10457-10463.

89. Heltweg B, et al. Antitumor activity of a small-molecule inhibitor of human silent information regulator 2 enzymes. Cancer Res. 2006;66(8):4368-4377.

90. Ota $\mathrm{H}$, et al. Sirt1 inhibitor, Sirtinol, induces senescence-like growth arrest with attenuated RasMAPK signaling in human cancer cells. Oncogene. 2006;25(2):176-185

91. Lain S, et al. Discovery, in vivo activity, and mechanism of action of a small-molecule p53 activator. Cancer Cell. 2008;13(5):454-463.

92. Suzuki K, et al. SRT1720, a SIRT1 activator, promotes tumor cell migration, and lung metastasis of breast cancer in mice. Oncology reports. 2012; 27(6):1726-1732.

93. Fang Y, Nicholl MB. Sirtuin 1 in malignant transformation: friend or foe? Cancer Lett. 2011;306(1):10-14.

94. Wang RH, et al. Interplay among BRCA1, SIRT1, and Survivin during BRCA1-associated tumorigenesis. Mol Cell. 2008;32(1):11-20. 
95. Lim JH, Lee YM, Chun YS, Chen J, Kim JE, Park JW. Sirtuin 1 modulates cellular responses to hypoxia by deacetylating hypoxia-inducible factor 1 alpha. Mol Cell. 2010;38(6):864-878.

96. Firestein R, et al. The SIRT1 deacetylase suppresses intestinal tumorigenesis and colon cancer growth. PloS One. 2008;3(4):e2020.

97. Wang RH, et al. Impaired DNA damage response, genome instability, and tumorigenesis in SIRT1 mutant mice. Cancer Cell. 2008;14(4):312-323.

98. Herranz D, et al. Sirt 1 improves healthy ageing and protects from metabolic syndrome-associated cancer. Nat Commun. 2010;1:3.

99. North BJ, Verdin E. Mitotic regulation of SIRT2 by cyclin-dependent kinase 1-dependent phosphorylation. J Biol Chem. 2007;282(27):19546-19555.

100. Pandithage R, et al. The regulation of SIRT2 function by cyclin-dependent kinases affects cell motility. J Cell Biol. 2008;180(5):915-929.

101.Dryden SC, Nahhas FA, Nowak JE, Goustin AS, Tainsky MA. Role for human SIRT2 NAD-dependent deacetylase activity in control of mitotic exit in the cell cycle. Mol Cell Biol. 2003;23(9):3173-3185.

102.Das C, Lucia MS, Hansen KC, Tyler JK. CBP/ p300-mediated acetylation of histone $\mathrm{H} 3$ on lysine 56. Nature. 2009;459(7243):113-117.

103. Vaquero A, et al. SirT2 is a histone deacetylase with preference for histone $\mathrm{H} 4$ Lys 16 during mitosis. Genes Dev. 2006;20(10):1256-1261.

104. Kim HS, et al. SIRT2 maintains genome integrity and suppresses tumorigenesis through regulating APC/C activity. Cancer Cell. 2011;20(4):487-499.

105. Jin $\mathrm{YH}$, et al. Sirt2 interacts with 14-3-3 beta/gamma and down-regulates the activity of p53. Biochem Biophys Res Commun. 2008;368(3):690-695.

106. Haigis MC, Deng CX, Finley LW, Kim HS, Gius
D. SIRT3 is a mitochondrial tumor suppressor: a scientific tale that connects aberrant cellular ROS, the Warburg effect, and carcinogenesis. Cancer Res. 2012;72(10):2468-2472.

107. Tao R, et al. Sirt3-mediated deacetylation of evolutionarily conserved lysine 122 regulates MnSOD activity in response to stress. Mol Cell. 2010; 40(6):893-904.

108. Qiu X, Brown K, Hirschey MD, Verdin E, Chen D. Calorie restriction reduces oxidative stress by SIRT3-mediated SOD2 activation. Cell Metab. 2010; 12(6):662-667.

109. Finley LW, et al. SIRT3 opposes reprogramming of cancer cell metabolism through HIF1alpha destabilization. Cancer Cell. 2011;19(3):416-428.

110.Alhazzazi TY, Kamarajan P, Verdin E, Kapila YL. SIRT3 and cancer: tumor promoter or suppressor? Biochim Biophys Acta. 2011;1816(1):80-88.

111. Mao Z, et al. SIRT6 promotes DNA repair under stress by activating PARP1. Science. 2011; 332(6036):1443-1446.

112. Barber MF, et al. SIRT7 links H3K18 deacetylation to maintenance of oncogenic transformation. Nature. 2012;487(7405):114-118.

113. Seligson DB, et al. Global levels of histone modifications predict prognosis in different cancers. Am J Pathol. 2009;174(5):1619-1628.

114.Donmez G, Wang D, Cohen DE, Guarente L. SIRT1 suppresses beta-amyloid production by activating the alpha-secretase gene ADAM10. Cell. 2010; 142(2):320-332.

115. Jeong H, et al. Sirt1 mediates neuroprotection from mutant huntingtin by activation of the TORC1 and CREB transcriptional pathway. Nat Med. 2012; 18(1):159-165.

116. Jiang M, et al. Neuroprotective role of Sirt1 in mam- malian models of Huntington's disease through activation of multiple Sirt1 targets. Nat Med. 2012;18(1):153-158.

117. Donmez G, Arun A, Chung CY, McLean PJ, Lindquist S, Guarente L. SIRT1 protects against alpha-synuclein aggregation by activating molecular chaperones. J Neurosci. 2012;32(1):124-132.

118.Sasaki Y, Araki T, Milbrandt J. Stimulation of nicotinamide adenine dinucleotide biosynthetic pathways delays axonal degeneration after axotomy. J Neurosci. 2006;26(33):8484-8491.

119. Araki T, Sasaki Y, Milbrandt J. Increased nuclear NAD biosynthesis and SIRT1 activation prevent axonal degeneration. Science. 2004;305(5686):1010-1013.

120.Pallos J, et al. Inhibition of specific HDACs and sirtuins suppresses pathogenesis in a Drosophila model of Huntington's disease. Hum Mol Genet. 2008;17(23):3767-3775.

121.Luthi-Carter R, et al. SIRT2 inhibition achieves neuroprotection by decreasing sterol biosynthesis. Proc Natl Acad Sci U S A. 2010;107(17):7927-7932.

122. Outeiro TF, et al. Sirtuin 2 inhibitors rescue alphasynuclein-mediated toxicity in models of Parkinson's disease. Science. 2007;317(5837):516-519.

123. Bobrowska A, Donmez G, Weiss A, Guarente L, Bates G. SIRT2 ablation has no effect on tubulin acetylation in brain, cholesterol biosynthesis or the progression of Huntington's disease phenotypes in vivo. PLoS One. 2012;7(4):e34805.

124. Beirowski B, et al. Sir-two-homolog 2 (Sirt2) modulates peripheral myelination through polarity protein Par-3/atypical protein kinase C (aPKC) signaling. Proc Natl Acad Sci US A. 2011;108(43):E952-E961.

125. Someya S, et al. Sirt3 mediates reduction of oxidative damage and prevention of age-related hearing loss under caloric restriction. Cell. 2010;143(5):802-812. 